\title{
A new sdO+dM binary with extreme eclipses and reflection effect
}

\author{
A. Derekas ${ }^{1,2}$, P. Németh ${ }^{3}$ J. Southworth ${ }^{4}$, T. Borkovits ${ }^{5,1}$, K. Sárneczky ${ }^{2}$, A. Pál ${ }^{2}$, B. \\ Csák $^{1}$, D. Garcia-Alvarez ${ }^{6,7,8}$, P. F. L. Maxted ${ }^{4}$, L. L. Kiss ${ }^{2,9}$, K. Vida ${ }^{2}$, Gy. M. Szabó ${ }^{1,2}$, \\ L. Kriskovics ${ }^{2}$
}

\begin{abstract}
We report the discovery of a new totally-eclipsing binary $\left(\mathrm{RA}=06^{\mathrm{h}} 40^{\mathrm{m}} 29^{\mathrm{s}} 11\right.$; $\left.\mathrm{DEC}=+38^{\circ} 56^{\prime} 52^{\prime \prime} 2 ; \mathrm{J}=2000.0 ; \mathrm{R}_{\max }=17.2 \mathrm{mag}\right)$ with an sdO primary and a strongly irradiated red dwarf companion. It has an orbital period of $P_{\text {orb }}=0.187284394(11) \mathrm{d}$ and an optical eclipse depth in excess of 5 magnitudes. We obtained two low-resolution classification spectra with GTC/OSIRIS and ten medium-resolution spectra with WHT/ISIS to constrain the properties of the binary members. The spectra are dominated by $\mathrm{H}$ Balmer and HeII absorption lines from the sdO star, and phase-dependent emission lines from the irradiated companion. A combined spectroscopic and light curve analysis implies a hot subdwarf temperature of $T_{\text {eff }}(\mathrm{spec})=55000 \pm 3000 \mathrm{~K}$, surface gravity of $\log g(\mathrm{phot})=6.2 \pm 0.04$ (cgs) and a He abundance of $\log (n \mathrm{He} / n \mathrm{H})=-2.24 \pm 0.40$. The hot sdO star irradiates the red-dwarf companion, heating its substellar point to about $22500 \mathrm{~K}$. Surface parameters for the companion are difficult to constrain from the currently available data: the most remarkable features are the strong $\mathrm{H}$ Balmer and C II-III lines in emission. Radial velocity estimates are consistent with the $\mathrm{sdO}+\mathrm{dM}$ classification. The photometric data do not show any indication of sdO pulsations with amplitudes greater than $7 \mathrm{mmag}$, and $\mathrm{H} \alpha$-filter images do not provide evidence of the presence of a planetary nebula associated with the sdO star.
\end{abstract}

Subject headings: binaries: eclipsing — stars: fundamental parameters — stars: low-mass — subdwarfs

\footnotetext{
${ }^{1}$ ELTE Gothard Astrophysical Observatory, H-9704 Szombathely, Szent Imre herceg út 112, Hungary; E-mail: derekas@gothard.hu

${ }^{2}$ Konkoly Observatory, Research Centre for Astronomy and Earth Sciences, Hungarian Academy of Sciences, H1121

${ }^{3}$ Dr. Karl Remeis-Observatory \& ECAP, Astronomisches Inst., FAU Erlangen-Nuremberg, 96049 Bamberg, Germany

${ }^{4}$ Astrophysics Group, Keele University, Newcastleunder-Lyme, ST5 5BG, UK

${ }^{5}$ Baja Astronomical Observatory of Szeged University, H-6500 Baja, Szegedi út, Kt. 766, Hungary

${ }^{6}$ Instituto de Astrofísica de Canarias, E-38205 La Laguna, Tenerife, Spain

${ }^{7}$ Dpto. de Astrofísica, Universidad de La Laguna, 38206 La Laguna, Tenerife, Spain

${ }^{8}$ Grantecan CALP, 38712, Breña Baja, La Palma, Spain

${ }^{9}$ Sydney Institute for Astronomy, School of Physics, University of Sydney, Australia
}

\section{Introduction}

Hot subdwarf stars are located between the upper main sequence and the white dwarf (WD) sequence in the Hertzsprung-Russell diagram. They are evolved, core-helium burning, low-mass stars $\left(M \approx 0.5 M_{\odot}\right)$ with very thin hydrogen envelopes (Heber 2009). Among hot subdwarfs, sdO stars $\left(T_{\text {eff }}>38000 K\right)$ represent a significantly smaller fraction than sdBs $\left(T_{\text {eff }}<35000 K\right)$. Spectroscopically, sdO stars show a large variety: the two main groups are the H-rich ( $\mathrm{sdO}$ ) and He-rich (He-sdO) stars. Stroeer et al. (2007) showed that a strong correlation exists among surface temperature, $\mathrm{He}$ and $\mathrm{C}, \mathrm{N}$ abundances in He-sdO stars.

Canonical stellar evolution theory predicts that sdO stars evolve from sdB stars. Whilst binarity is quite frequent among sdBs, with a binary fraction of about 50\% (Maxted et al. 2001; Napiwotzki et al. 2004), the fraction of binary 
He-sdOs is very low Kawka et al. 2015). Many sdO binaries are associated with a planetary nebula (PN), like UU Sge, V477 Lyr and BE UMa (Pollacco \& Bell 1994; Pollacco, Bell \& Hilditch 1994; Afșar \& İbanoğlu 2008). The sdO stars in these binaries are hotter and more massive stars in the immediate post giant branch stage. Binaries with compact $\mathrm{sdO}$ stars evolve from $\mathrm{sdB}$ binaries and the $\sim 120$ Myr sdB lifetime is long enough for their PNe to attenuate and become hardly detectable (Aller et al. 2015).

Han et al. (2002, 2003) performed binary population syntheses and identified several evolutionary channels that lead to the formation of hot subdwarf stars. In close binaries that evolve through one or more common envelope (CE) phases (Paczynski 1976), the secondary is engulfed by the atmosphere of the primary while it is on the red giant branch.

As the secondary spirals inward due to tidal friction, the red giant loses mass. By the end of the CE phase the primary loses most of its envelope and the binary orbital period shrinks to a few hours. If the core gained enough mass for He ignition during the preceding evolution the primary experiences a He-flash and settles on the extreme horizontal branch. In case the core mass is insufficient for He burning, the primary evolves as a low-mass pre-white dwarf. In both cases the common envelope is ejected during the final stage of CE evolution and a very close binary remains (Taam \& Ricker 2006). While this theory can explain the mass loss required for the formation of hot subdwarfs, it also needs a precise timing between mass loss and the core helium flash. Eclipsing hot subdwarf binaries with irradiated companions can give insight into the details of these processes, making such binaries fundamental to understand CE evolution.

Here we report the discovery of a new eclipsing binary with an sdO primary and a strongly irradiated red dwarf companion. After describing the observations, we discuss the spectral modelling and determination of the stellar and orbital parameters from the spectroscopic and light curve analysis.

\section{Observations}

Konkoly J064029.1 $+385652.2\left(\mathrm{RA}=06^{\mathrm{h}} 40^{\mathrm{m}} 29^{\mathrm{s}} 11 ;\right.$ $\mathrm{DEC}=+38^{\circ} 56^{\prime} 52^{\prime \prime} 2 ; \mathrm{J}=2000.0 ; \mathrm{R}_{\max }=17.2 \mathrm{mag}$; hereafter J0640+3856) was discovered serendipitously during regular astrometric observations of minor planets. On one of the images the object completely disappeared, suggesting a sudden deep eclipse. We started to monitor J0640+3856 using the 0.6/0.9/1.8 m Schmidt telescope at Piszkéstető Observatory. We took CCD photometric observations on seven nights between December 2013 and February 2014 using Johnson/Bessell $V$ and Cousins $R_{\mathrm{C}}$ filters, and also without filters. The telescope was equipped with an Apogee ALTA-U $4 \mathrm{k} \times 4 \mathrm{k}$ CCD camera. The observations revealed that the period is $0.187 \mathrm{~d}$ and there is a strong reflection effect with an amplitude of $\sim 0.5 \mathrm{mag}$. The sudden and deep primary minimum suggested that the system might contain a hot and small primary star eclipsed by a cool secondary object.

We obtained further observations via the service program on the $4.2 \mathrm{~m}$ William Herschel Telescope (WHT) on La Palma. In February and March 2014 we observed two primary eclipses with Sloan $r^{\prime}$ and $i^{\prime}$ filters and a secondary eclipse in $i^{\prime}$ band, using the ACAM imager (Benn, Dee \& Agócs 2008). The data were reduced with standard procedures. The flat minima indicated that the eclipse depths are 6 and $5 \mathrm{mag}$, respectively, and the totality of the primary eclipse lasts for about $5.7 \mathrm{~min}$, while the eclipse duration is approximately $24 \mathrm{~min}$.

On March 1, 2014, we obtained two spectra using the $10.4 \mathrm{~m}$ Gran Telescopio Canarias (GTC) and OSIRIS spectrograph 11 on La Palma. Due to the highly variable seeing we used a 1.23 arcsec slit providing a dispersion of $\Delta \lambda \approx 1.6 \AA /$ pixel and $\Delta \lambda \approx 4.2 \AA /$ pixel with the $\mathrm{R} 2500 \mathrm{~V}$ and the R1000B grisms, respectively. These low- and medium-resolution spectra were taken between 3850-7400 $\AA$ and 4400-6000 $\AA$, and reached a signal-to-noise ratio (SNR) $\sim 30$ with 540 s exposure times.

We acquired phase-resolved spectroscopy on March 6, 2014, using the WHT and the dual-beam ISIS spectrograph 2 . This was operated with the

\footnotetext{
${ }^{1}$ http://www.gtc.iac.es/instruments/osiris

${ }^{2}$ http://www.ing.iac.es/Astronomy/instruments/isis
} 
R600B/R600R gratings and a 1 arcsec slit, providing a resolution of $\Delta \lambda=1 \AA /$ pixel in the blue and covering the $3800-5200 \AA$ and $6200-7800 \AA$ regions. Since the $H \alpha$ line was weak, we did not use the red region in the analysis. We took ten spectra between orbital phases $\varphi=0.47$ and 0.82 with $600 \mathrm{~s}$ exposure times. The average SNR of these spectra is 25 .

All spectroscopic data were reduced with our IRAF based data reduction pipeline. Bias and flat field corrections were done with the CCDPROC task, raw spectra and arc calibrations were extracted with APALL and wavelength calibrated with the IDENTIFY/REIDENTIFY tasks. We identified $\sim 150 \mathrm{CuArNe}$ lines in the WHT calibration data, that allowed for an eighth order Legendre polynomial dispersion function with a rootmean-square $(\mathrm{RMS}) \sim 0.1 \AA$. The GTC HgArNeXe calibrations allowed us to identify $\sim 50-100$ lines and use a sixth order dispersion function with $\mathrm{RMS} \sim 0.6 \AA$.

In April 2014 we obtained 1.5 h CCD photometry with the $1 \mathrm{~m}$ RCC telescope at the Piszkésteto" Observatory, using an FLI camera with a field of view of $9.4^{\prime} \times 9.4^{\prime}$, with a Sloan $r^{\prime}$ filter and $2 \times 2$ binning giving a plate scale of $0.27^{\prime \prime} /$ pixel. In addition, we obtained $\sim 21 \mathrm{~h}$ of fast photometry ( $5 \mathrm{~s}$ exposure times) in December 2014 and February 2015 using the same telescope with the OCELOT camera (an Andor iXon+888 EMCDD camera) without filter, in order to search for any oscillations from the sdO star. We did not detect any significant periodic signal with amplitudes greater than 7 mmag.

In order to detect whether any PN could be associated with the sdO, we took $27 \mathrm{H} \alpha$-filter images with 120 s exposure times with the same telescope in April 2014. The composite image did not show any sign of a PN.

\section{The orbital period}

We checked the stability of the orbital period. In order to determine accurate times of minima the WHT light curves of the two primary and one secondary minima were used as templates, and these template curves were fitted to the other, less accurate light curves as described in Borkovits et al. (2015) in details. Thus, we obtained 13 primary and 14 secondary times of min- ima, which are listed in Table 1] and plotted in Fig. 1. For the period variation analysis, however, only the primary minima were used, with the one exception of the outlying point at JD 2456691. Therefore, we used twelve primary minima obtained between JD 2456684 and JD 2457068 to calculate the following ephemeris:

$T_{\min , \mathrm{I}}=2456709.58464(2)+0 \mathrm{~d} 187284762(81) \times E$,

where the epoch is given in BJD (TDB). We did not find any evidence for a variation in the period, however, it should be noted that the scattering of the individual points exceeds significantly the estimated statistical errors.

\section{Spectroscopy}

\subsection{Modelling the composite spectrum}

The first light curve of J0640+3856 clearly suggested a HW Vir type eclipsing binary with a strongly irradiated cool companion. In such binaries the orbit is small and the companion is relatively large to produce a prominent reflection effect in the light curve and provide full eclipses of the primary.

Our classification spectra showed strong emission lines of $\mathrm{C}$ superimposed on the shallow absorption spectrum of a hot star. J0640+3856 is therefore a double lined spectroscopic binary, making it a target with high potential. Disentangling the spectrum of the hot spot usually requires high-contrast high-SNR spectroscopy and a challenging analysis procedure, similar to direct exoplanet spectroscopy. The spectra of irradiated companions have been disentangled and analysed in several hot subdwarf binaries, e.g. EC 11575 and V664 Cas (Exter et al. 2005), WD0137-349 (Maxted et al. 2006) and AA Dor (Vučković et al. 2008). However, these analyses were limited to relative line strength and radial velocity measurements based on Gaussian line profiles. Although grids of irradiated M-dwarf spectra are available (e.g. Barman et al. 2004), their application to fit observed data is limited. Wawrzyn et al. (2009) presented a self-consistent model atmosphere analysis for the hot subdwarf binary UU Sge. Here we follow their methods based on the description in Günther \& Wawrzyn (2011).

J0640+3856 showed a double lined composite spectrum immediately in low-resolution moderate- 
Table 1: Times of minima of J0640+3856.

\begin{tabular}{|c|c|c|c|c|c|c|c|}
\hline \multicolumn{4}{|c|}{ Primary minima } & \multicolumn{4}{|c|}{ Secondary minima } \\
\hline $\begin{array}{l}\text { BJD (TDB) } \\
-2400000\end{array}$ & $\begin{array}{r}\text { Cycle } \\
\text { no. }\end{array}$ & $\begin{array}{l}\text { std. dev. } \\
(d)\end{array}$ & instrument & $\begin{array}{l}\text { BJD (TDB) } \\
-2400000\end{array}$ & $\begin{array}{r}\text { Cycle } \\
\text { no. }\end{array}$ & $\begin{array}{l}\text { std. dev. } \\
(d)\end{array}$ & instrument \\
\hline 56684.301289 & -135.0 & 0.000004 & Schmidt $\mathrm{R}_{C}$ & 56654.616610 & -293.5 & 0.000019 & Schmidt unfilt. \\
\hline 56684.488447 & -134.0 & 0.000004 & Schmidt $\mathrm{R}_{C}$ & 56657.424647 & -278.5 & 0.000019 & Schmidt unfilt. \\
\hline $56691.417455^{*}$ & -97.0 & 0.000031 & Schmidt V & 56684.394740 & -134.5 & 0.000019 & Schmidt $\mathrm{R}_{C}$ \\
\hline 56692.354351 & -92.0 & 0.000004 & Schmidt V & 56689.265080 & -108.5 & 0.000032 & Schmidt V \\
\hline 56693.290882 & -87.0 & 0.000004 & Schmidt $\mathrm{R}_{C}$ & 56691.325691 & -97.5 & 0.000007 & Schmidt V \\
\hline 56695.350946 & -76.0 & 0.000001 & WHT r' & 56691.509921 & -96.5 & 0.000017 & Schmidt V \\
\hline 56709.584600 & 0.0 & 0.000002 & WHT i' & 56692.260600 & -92.5 & 0.000019 & Schmidt V \\
\hline 56775.321665 & 351.0 & 0.000021 & $\mathrm{RCC} \mathrm{r}^{\prime}$ & 56692.448527 & -91.5 & 0.000024 & Schmidt V \\
\hline 57022.537442 & 1671.0 & 0.000005 & RCC unfilt. & 56693.385087 & -86.5 & 0.000023 & Schmidt $\mathrm{R}_{C}$ \\
\hline 57067.298585 & 1910.0 & 0.000002 & RCC unfilt. & 56729.530347 & 106.5 & 0.000016 & WHT i' \\
\hline 57067.485826 & 1911.0 & 0.000003 & RCC unfilt. & 57022.631307 & 1671.5 & 0.000021 & RCC unfilt. \\
\hline 57068.234937 & 1915.0 & 0.000003 & RCC unfilt. & 57067.391972 & 1910.5 & 0.000015 & RCC unfilt. \\
\hline 57068.422263 & 1916.0 & 0.000002 & RCC unfilt. & 57068.328437 & 1915.5 & 0.000013 & RCC unfilt. \\
\hline & & & & 57068.515991 & 1916.5 & 0.000018 & RCC unfilt. \\
\hline
\end{tabular}

Note. Secondary minima, and the one primary minima denoted by $*$ were not included in the period study.

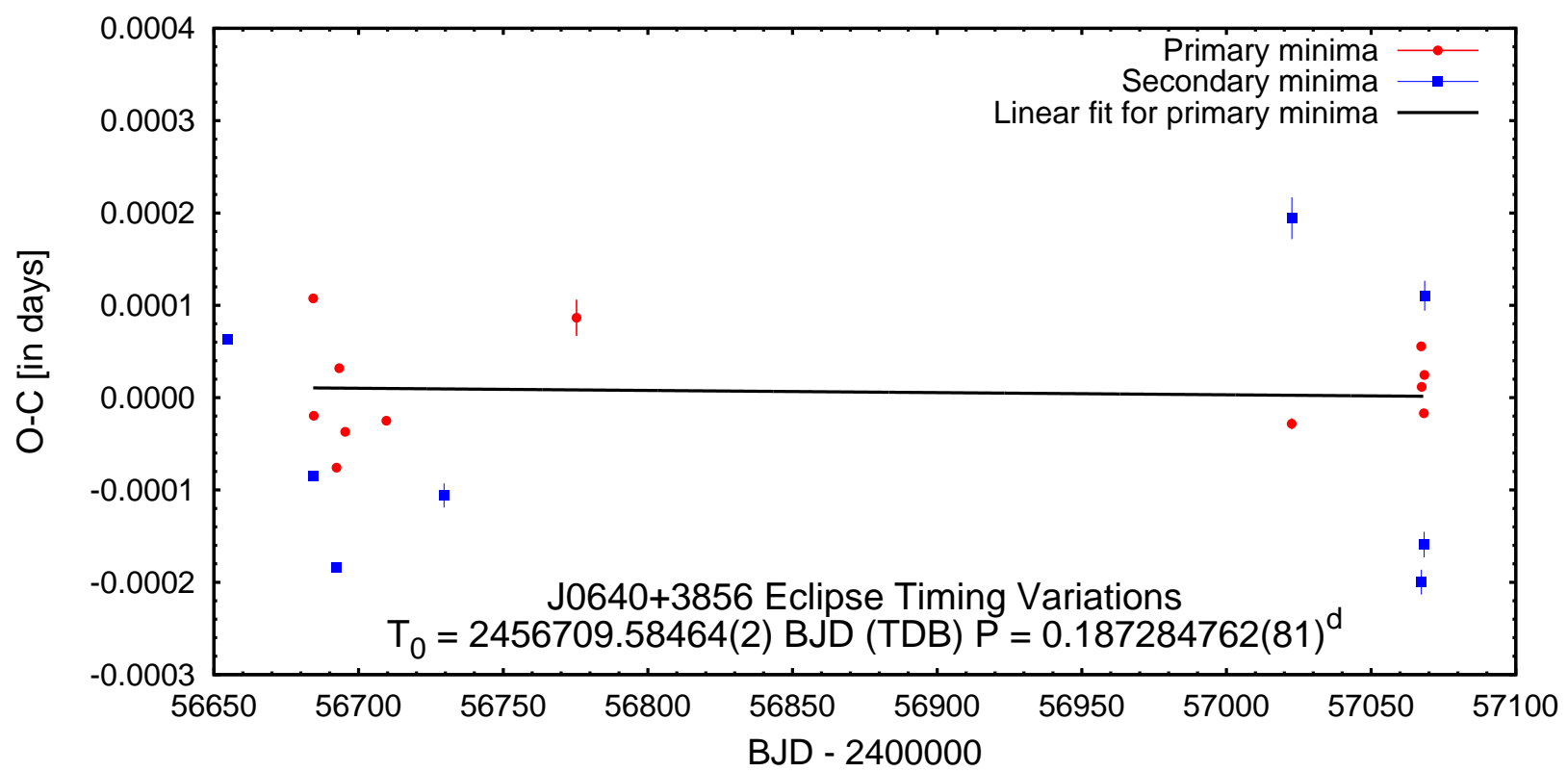

Fig. 1. - Eclipse Timing Variation diagram of J0640+3856. Red circles represent the primary and blue boxes the secondary minima. For a better visibility, secondary minima with a large scatter are not shown here. The black line represents the linear fit for the primary minima, which we used to calculate the ephemeris and orbital period given both in the text and the figure. It shows that the period is well determined and constant over one year. 


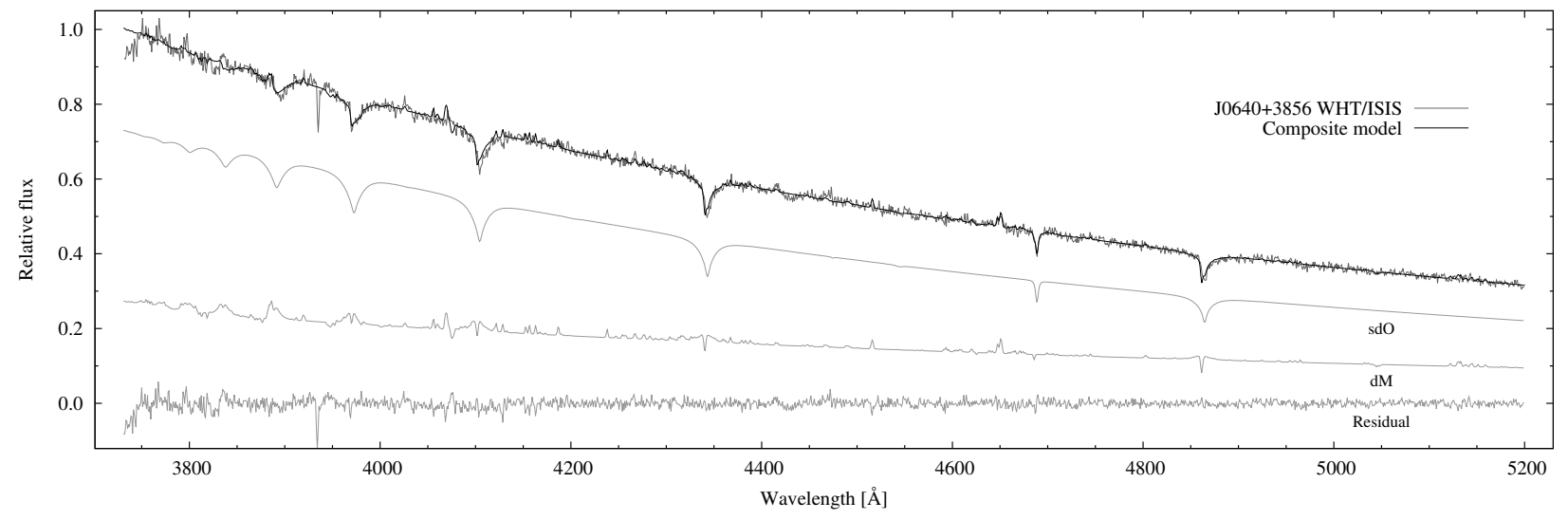

Fig. 2. - Spectral decomposition of the WHT/ISIS spectrum at $\varphi=0.647$. The spectral features can be described with the superposition of a $55000 \mathrm{~K}$ sdO star and an irradiated dM companion heated to $\sim 22500 \mathrm{~K}$.

SNR spectroscopy, as achievable with 4 m-class telescopes, despite being fainter by $\sim 6$ magnitudes in $V$ than AA Dor. This suggests that both components are more extreme: the sdO star must be hotter and the companion must be a larger, probably earlier-M star that suffers a stronger irradiation. The most remarkable spectral features are the distorted, phase-dependent $\mathrm{H}$ Balmer line profiles, the strong He II absorption indicating an sdO star and C II-III lines in emission from the irradiated companion. These features defined the starting models in our analysis. The WHT observations include the secondary minimum, what makes them ideal to investigate the irradiated hemisphere of the companion.

Our spectral analysis was based on the steepestdescent iterative binary fit procedure, XTGRID, developed by Németh et al. (2012). XTGRID employs Tlusty/SynsPeC (Hubeny \& Lanz 1995; Lanz \& Hubeny 2003) non-LTE model atmospheres and synthetic spectra to reproduce composite binary spectra. The strong irradiation allowed us to model both components in J0640+3856 with Tlusty. The model atmospheres include $\mathrm{H}, \mathrm{He}, \mathrm{C}, \mathrm{N}$ and $\mathrm{O}$ opacities consistently in the atmosphere structure and synthetic spectrum calculations for both stars. Although we could not identify CNO absorption lines in the sdO star and kept their abundances fixed at $\log (n \mathrm{X} / n \mathrm{H})=-6$, we included $\mathrm{CNO}$ opacities, because they have an effect on the temperature structure of non-LTE atmosphere models (Werner 1996).
We started an XTGRID process for the sdO star and another for an $\mathrm{M}$ dwarf that is irradiated by the sdO. These processes iteratively updated the stellar parameters and fit the observed composite spectrum together. As a first approximation we applied an isotropically irradiated model for the inner hemisphere of the companion. We assumed a black-body energy distribution of the irradiating flux with appropriate temperature for the sdO. The geometric dilution factor $(W)$ is Mihalas 1978):

$$
W=\frac{1}{2}\left(1-\sqrt{1-\left(\frac{\mathrm{R}_{2}}{a}\right)^{2}}\right),
$$

where $R_{2}$ is the radius of the secondary and $a$ is the semi-major axis. The dilution factor describes the strength of irradiation. We used an empirical dilution factor $W_{e}=0.05$ that was found for the substellar point in the similar system AA Dor. The empirical dilution factor is a fudge factor in our analysis and substantially larger than the geometrical dilution factor. The difference is probably due to the non-planckian sdO spectrum and due to the fact that the spectrum of the irradiated companion cannot be fully described with the conditions in the substellar point, as suggested by Günther \& Wawrzyn (2011).

The combined spectrum at $\varphi=0.647$ has a SNR of $\sim 45$, therefore we modelled this spectrum as shown in Fig. 2. The flux contribution of the hot spot was determined for this spectrum 

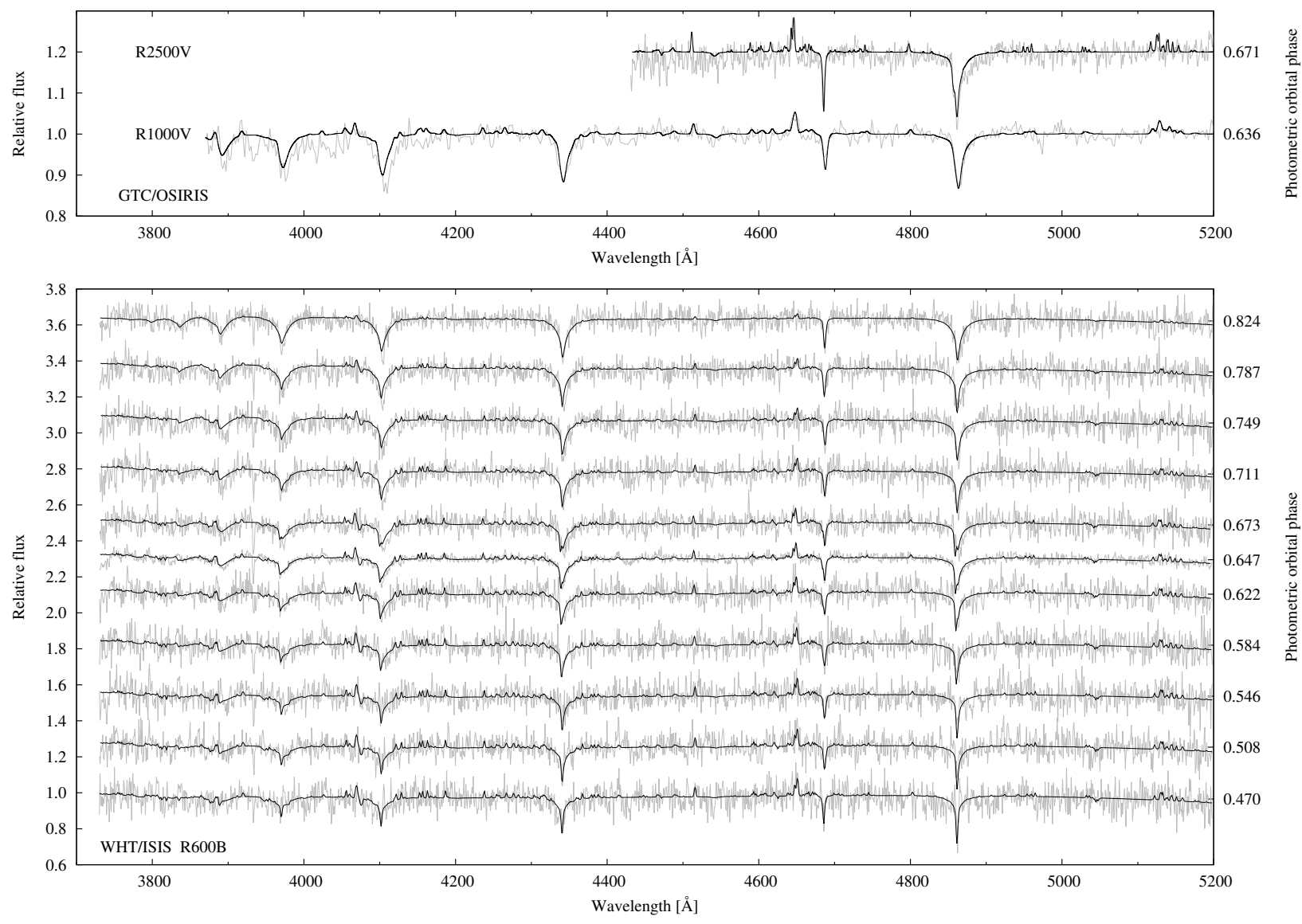

Fig. 3. - Top: GTC/OSIRIS spectra of J0640+3856 (grey) with best fit models (black). Bottom: Best fit models (black) for the phase-dependent radial velocity corrected composite spectra obtained with WHT/ISIS (grey). We applied a spectral decomposition of the middle spectrum at orbital phase $\varphi=0.647$. All the other models were calculated from this decomposition by shifting the components in radial velocity and scaling them by the orbital phase to account for the visibility of the substellar point of the companion. 
and we approximated it for the other spectra by scaling with orbital phase according to $F_{\mathrm{dM}} / F_{\mathrm{sd}} \sim$ $0.43 \sin ^{2}(\pi \varphi)$. The TLusty model provides the temperature structure of the irradiated companion. The temperature decreases steeply inward, reaches a minimum and progressively increases again. The minimum temperature is $T=22500 \mathrm{~K}$ at optical depth $\tau \approx 0.6$, which we associate with the photospheric effective temperature of the substellar point. The parameters derived from the spectroscopic modelling are listed in Table 2 and suggest an sdO+dM binary.

Next we shifted the components in velocity space to reproduce the phase-dependent composite spectra. Fig. 3 shows the best fits for the ten WHT blue arm observations. These fits show that all $\mathrm{H}$ line profiles are contaminated by the emission lines from the companion and the relative strength of this contamination increases towards the Balmer series limit. The inverted temperature structure of the companion results in high excitation lines compared to the photospheric temperature. Such emission lines of C III and Si III have been observed from the irradiated companion in other systems (e.g.: V477 Lyr, AA Dor). Therefore, a He II $4686 \AA$ emission line may be expected as well. However, this line forms between very high lying levels in the $\mathrm{He}$ ion and at the low $\mathrm{He}$ abundance of J0640+3856 we did not find any contribution. The fact that the He II $4686 \AA$ line forms exclusively in the sdO star allowed us to measure its radial velocity. The $\mathrm{C}$ II $4267 \AA$ line, the $\mathrm{C}$ III blend near $4650 \AA$ and $4070 \AA$ are in emission and come from the irradiated companion. Based on a selection of these lines we could also estimate the radial velocity of the companion.

\subsection{Radial velocity and stellar masses}

Even though precision radial velocity measurements would require high-dispersion and higherSNR spectra, we attempted to estimate radial velocities from the WHT/ISIS spectra, because the amplitudes put a useful constraint on the mass ratio. First, we checked the dispersion correction, which was based on 150 lines of the CuAr and $\mathrm{CuNe}$ calibration lamps, and tested the stability on the CaII K line. This line forms mostly in the interstellar medium and therefore its radial velocity can be used to check dispersion offsets. We found the $\mathrm{K}$ line to be consistent in all our spec- tra, but systematically offset by $40 \mathrm{~km} \mathrm{~s}^{-1}$. Most of this offset can be explained with the barycentric velocity correction $v_{B C}=-26.5 \mathrm{~km} \mathrm{~s}^{-1}$ during the observations. The interstellar extinction towards J0640+3856 is $E(\mathrm{~B}-\mathrm{V})=0.124 \pm 0.005$ (Schlafly \& Finkbeiner 2011), therefore we associate the remaining radial velocity shift of the Ca II K line with the relative velocity of the interstellar material with respect to the Solar system. We performed two independent experiments to measure velocities and limited our measurements to the He II $4686 \AA$ line for the sdO and to the $\mathrm{C}$ III blend between $4647-4652 \AA$ for the $\mathrm{dM}$ companion as these are the strongest undistorted features in the spectra.

In the first method we combined each consecutive spectrum, like those at $\varphi=0.622$ and 0.673 to get the one at 0.647 in Fig. 3. and applied a Savitzky-Golay filter (Savitzky \& Golav 1964). Then we measured the SNR of each of these combined spectra. Next, we determined the radial velocity with an iterative chi-square minimization cross-correlation procedure. We decided to use the preceding spectrum as a self-template to reduce systematic effects in the chi-square method. For each observed spectrum we performed 100 radial velocity measurements to obtain a mean value and standard deviation. We resampled the spectra according to the SNR before each individual measurement.

The second method was based on visual inspection. This allowed us to compare the observations to synthetic spectra directly and derive absolute radial velocities.

We found a good match between the two methods for the sdO star. However, for the dM companion we found that the cross-correlation method underestimated the radial velocity by about $40 \%$. The most plausible reason for this is orbital smearing that makes the spectral lines considerably shallower and broader, while the decreasing contribution of the companion also makes the last data points less reliable. Similarly for the sdO star, the last data points show a decreasing radial velocity in conflict with the photometric orbital period. We attributed these inconsistencies to a lower data quality and disregarded the last three spectra in the radial velocity measurement. The symmetric appearance of the reflection effect and secondary minimum suggest a circular orbit and 
a co-rotating companion. Assuming synchronised rotation we found a projected rotational velocity $v_{\text {rot }} \sin i=49.2 \mathrm{~km} \mathrm{~s}^{-1}$ using $R_{2}=0.2 \mathrm{R}_{\odot}$ from the light curve solution (see Sect. 5). As the centre of mass of the companion does not coincide with its centre of light we needed to correct the radial velocity with the projected rotational velocity at the centre of light. We calculated this correction as $-29 \mathrm{~km} \mathrm{~s}^{-1}$ at $\varphi=0.75$.

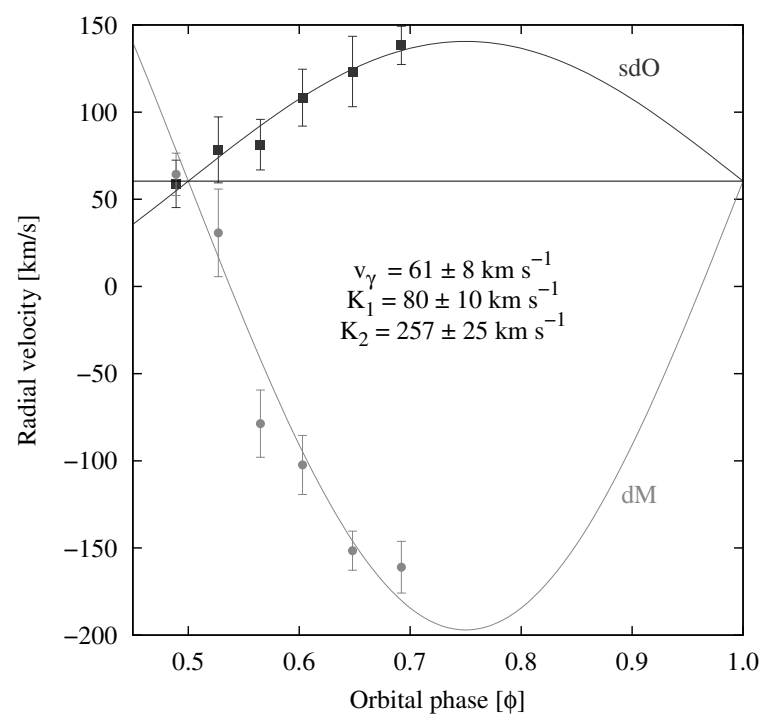

Fig. 4.- Radial velocity curves of the components of $\mathrm{J} 0640+3856$.

The six data points in Fig. 4 show a clear trend and suggest the corrected radial velocity semi-amplitudes: $K_{1}=80 \pm 10 \mathrm{~km} \mathrm{~s}^{-1}, K_{2}=$ $257 \pm 25 \mathrm{~km} \mathrm{~s}^{-1}$ and a barycentric system velocity $v_{\gamma}=61 \pm 8 \mathrm{~km} \mathrm{~s}^{-1}$. From the radial velocity semiamplitudes we found a mass ratio of $q=M_{2} / M_{1}=$ $K_{1} / K_{2}=0.31 \pm 0.04$. Assuming a circular orbit, the radial velocity semi-amplitudes define the projected semi-major axis $a \sin i \approx\left(K_{1}+K_{2}\right) P / 2 \pi$, where $P$ is the orbital period and $i$ is the inclination, which can be obtained from the eclipsing light curve solution. The observed orbital period and this semi-major axis define the total mass by Kepler's third law.

Using the inclination from the light curve solution $i=87.11^{\circ}$ and the radial velocity amplitudes the semi-major axis is $a=0.006 \mathrm{AU}(869000 \mathrm{~km})$. Then the total mass from Kepler's third law is $\mathrm{M}_{\text {tot }}=0.744 \mathrm{M}_{\odot}$.
According to our models the strong irradiation transforms the secondary atmosphere completely. The irradiated models are insensitive for the unperturbed temperature of the companion, therefore we cannot assess the night side temperature from the currently available irradiated spectrum. In turn, the models show that the Balmer emission lines are sensitive for the surface gravity (pressure) and to reproduce the line profile variations they require a $\log g>4.5$. Assuming that the mass of the subdwarf is close to the canonical $0.5 \mathrm{M}_{\odot}$, the measured surface gravity $\log g=4.9$ of the companion is most consistent with a mid-M type star.

\section{Light curve analysis}

We carried out a three-band simultaneous light curve analysis with the recently developed LightCURVEFACTORY code Borkovits et al. 2013, 2014). We chose the Sloan $i^{\prime}$ and $r^{\prime}$ light curves obtained using WHT/ACAM, which contain two flat primary minima offering strong geometrical constraints not only upon the inclination and the relative radii of the stars, but also, via Kepler's third law, the surface gravity of the stars given an approximate estimate of their mass. The third light curve used was the $R_{C^{-}}$ band dataset obtained with the Schmidt telescope at the Piszkéstető Observatory, which covers more than a full orbit of the system, including two primary minima. Despite a lower quality and the absence of measurements during the deepest parts of the primary minima, these data significantly improved the fit of the reflection effect and also contain useful information on the ellipsoidal variation or its absence that can be used to further constrain the mass ratio.

There is no indication for eccentricity from the spectroscopic orbit or the phase of secondary eclipse so we assumed that the orbit is circular, as expected for such a short-period binary star. Assuming a spherical primary, and a marginally oblated secondary star (which latter assumption was found to be reliable from a preliminary analysis of the eclipse geometry), it is a suitable approximation that the fractional radii of the components relate directly to the observable quantities of the full and totality-phase durations of the primary occultations. In such a way the whole system geometry was determined except for one free param- 


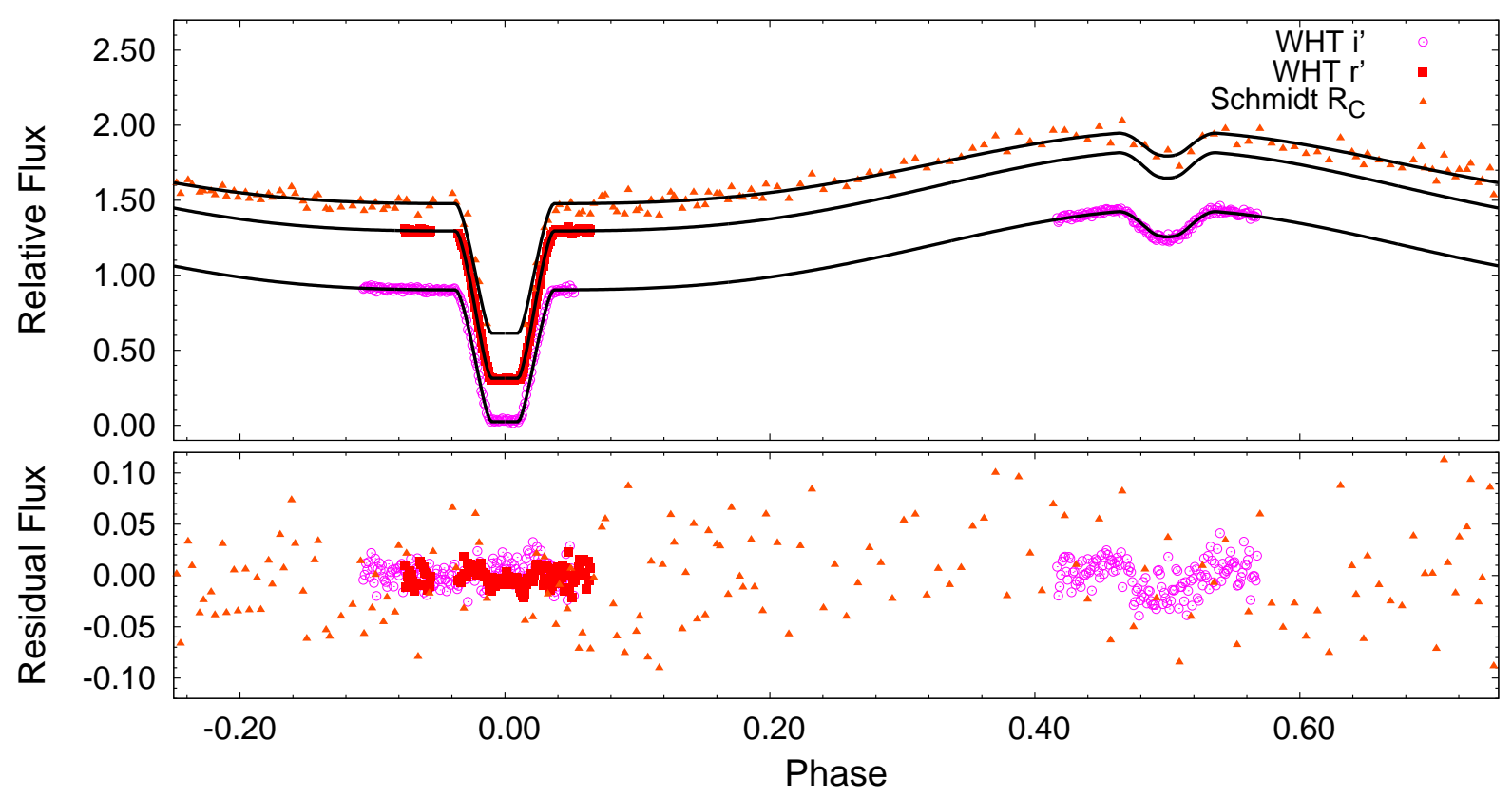

Fig. 5.- Observed light curves and solutions with their residuals for the Sloan $i^{\prime}, r^{\prime}(\mathrm{WHT})$ and $R_{C}$ (Piszkéstető Observatory) passband measurements.

eter, namely $i$. Therefore we were able to express the adjustable dynamical and geometrical parameters using the binary period $(P)$, epoch $\left(T_{0}\right)$, the orbital inclination $(i)$ and the mass ratio $(q)$. Although the mass ratio is also known from the radial velocity solution (see Sect.4.2), we made fits both with it freely adjusted and fixed to the spectroscopic value, as a consistency check. Due to the detached configuration and nearly spherical stellar shapes, the mass ratio has only a minor influence on our light curve solution.

Considering the atmospheric properties, the effective temperature of the sdO primary was fixed to the value obtained from the spectroscopic analysis $\left(T_{\text {eff } 1}=55000 \mathrm{~K}\right)$, while $T_{\text {eff2,d }}$ (night side of the secondary component) was the fifth adjusted parameter. The other atmospheric parameters limb darkening, gravity brightening coefficients, bolometric albedos and abundances of the sdO primary - were also kept fixed. For limb darkening we applied the logarithmic law, and the coefficients were calculated according to the passband- dependent precomputed tables 3 of the PHOEBE team (Prša \& Zwitter 2005; Prša 2011) which were based on the tables of Castelli \& Kurucz (2004).

On the other hand, most of the atmospheric parameters of the secondary star were involved into the fitting process. This was done because of the high irradiation which results in such a high temperature as $T=22500 \mathrm{~K}$ at the substellar point that there are significant deviations from LTE models as discussed e.g. in Barman et al. (2004). Amongst other consequences, this may change the full atmosphere to a radiative one, as was observed for other similar systems (e.g. AA Dor; Hilditch et al. 2003), and may even result in limb-brightening (i.e. negative limb darkening coefficients, see e.g. Pollacco \& Bell (1994), for V477 Lyr).

Finally, the passband luminosities of the primary component were also re-calculated in each step. A $\chi^{2}$ minimization was carried out using firstly the Levenberg-Marquardt differential corrections algorithm, and then a final refine-

${ }^{3}$ Downloaded from the site http://phoebe-project.org/1.0/ 
ment with a grid-search method. The resulting light curve solutions, and their residual curves are shown in Fig. 5 The values of the free parameters found from the best fit to the light curves are given in Table 2, together with the fixed parameters in the least-squares fit and some useful derived parameters.

The photometric mass ratio was found to be significantly smaller $(q=0.19 \pm 0.02)$ than the spectroscopic one. On the other hand, comparing the two solutions (with adjusted or fixed mass ratios), we found that all the other adjusted parameters, and also the $\chi^{2}$ have remained within their $1 \sigma$ values. This is a consequence of the small amplitude ellipsoidal variation, due to the minor oblateness of the secondary star and, therefore, the weak dependence of the light curve on $q$. We therefore decided to keep the solution obtained with the fixed spectroscopic mass ratio and the derived astrophysical quantities were computed accordingly. Considering the other quantities common to the spectroscopic and photometric analyses, while the light curve solution clearly confirms the spectroscopic temperature of the secondary, the local gravities were found to be higher.

Using the spectroscopic mass ratio we found a mass of $0.57 \pm 0.14 M_{\odot}$ for the sdO star and $0.18 \pm 0.05 M_{\odot}$ for the companion, in agreement with a mid-M dwarf (M6V) classification. We note that although the spectroscopic mass ratio is higher, yet consistent with our light curve analysis, the poor quality of our spectra means it is not precisely determined.

Comparing the spectroscopic and photometric $\log g$ values of the sdO primary, despite that the lower spectroscopic value is closer to expectations, the photometric result is evidently the more robust. This is because it is determined purely by the system geometry via the eclipse durations, which give the relative radii as a the function of $i$. Then, by the use of Kepler's third law, one can see that $g_{\text {pri }} \sim m_{\text {pri }}^{1 / 3} /(1+q)^{2 / 3}$. Therefore, even a $100 \%$ error in the mass of the primary would result in a 0.1 dex discrepancy in its $\log g$ value. This is valid only for spherical stars with negligible tidal and rotational effects, but our solution (i.e. the low values of the fractional radii) is consistent with the assumption that these effects play only a minor role in the system. As a consequence, we accept the high $\log g$ values obtained from the light curve analysis instead of their spectroscopic value and conclude that the sdO primary is a compact object on its way to the WD cooling sequence.

Turning to the adjusted atmospheric parameters of the secondary, its bolometric albedo $\left(A_{2}\right)$ was found to be greater than unity. This is not an unphysical solution, but implies that light from outside of a given photometric passband is being reprocessed and re-emitted in the passband. A similar situation has been found before for other systems (e.g., Southworth et al. 2011, for KIC 10661783, a totally eclipsing binary with a $\delta$ Scuti component and Almeida et al. 2012, for the eclipsing sdOB+dM binary V1828 Aql). We also found an unusually high gravity darkening coefficient $\left(\beta_{2}\right)$ for the secondary. The large uncertainty, however, makes this result ambigous. We note that the relatively poor fit to the data in secondary minimum is likely due to the simplistic treatment of the most highly-irradiated part of the atmosphere of the secondary component, which is eclipsed during secondary minimum. A complete physical description of the phsyics in this atmosphere is not available in our code, or in other commonly available light curve modelling codes.

\section{Summary and Conclusions}

We have discovered an sdO+M6V eclipsing binary $\left(\mathrm{RA}=06^{\mathrm{h}} 40^{\mathrm{m}} 29^{\mathrm{s}} 11 ; \quad \mathrm{DEC}=+38^{\circ} 56^{\prime} 52^{\prime \prime} 2\right.$; $\mathrm{J}=2000.0 ; \quad \mathrm{R}_{\max }=17.2 \mathrm{mag}$ ) that shows $6-\mathrm{mag}$ deep primary eclipses and a $\sim 0.5$ mag reflection effect. These are the most extreme variations among all HW Vir type binaries known to us and the primary minimum is even deeper than that of NN Ser, a well-known white dwarf with an extreme eclipse depth (Haefner et al. 2004). With photometric and spectroscopic follow-up we constrained the atmospheric properties of the components and the binary orbit. Although the specific spectral features and the effective temperature $\left(T_{\text {eff } 1}=55000\right.$ $\mathrm{K})$ classify the primary component as an sdO star, the surface gravity is at the upper limit of sdOs $(\log g=6.2 \mathrm{cgs})$ and the radius $\left(R=0.096 R_{\odot}\right)$ is smaller than for normal sdO stars. These parameters place the primary component in a special position, suggesting the sdO star is a pre-WD, similar to BE UMa which is classified as a borderline object between sdO subdwarfs and DAO white dwarfs (Ferguson et al. 1999). The non-detection 
Table 2: Stellar and orbital parameters derived from the spectroscopic and eclipsing light curve analysis.

\begin{tabular}{|c|c|c|}
\hline & \multicolumn{2}{|c|}{ orbital parameters } \\
\hline$\overline{P_{\text {orb }}(\text { days })}$ & \multicolumn{2}{|c|}{$0.18728550 \pm 0.00000005$} \\
\hline$T_{\text {MINI }}(\mathrm{BJD})$ & \multicolumn{2}{|c|}{$2456709.584565 \pm 0.000013$} \\
\hline$a^{d}\left(\mathrm{R}_{\odot}\right)$ & \multicolumn{2}{|c|}{$1.24857 \pm 0.09976$} \\
\hline$e$ & \multicolumn{2}{|c|}{0.0} \\
\hline$i\left({ }^{\circ}\right)$ & \multicolumn{2}{|c|}{$87.11 \pm 0.03$} \\
\hline$q_{\mathrm{spec}}^{d}$ & \multicolumn{2}{|c|}{$0.31 \pm 0.05$} \\
\hline$q_{\text {phot }}($ unused $)$ & \multicolumn{2}{|c|}{$0.19 \pm 0.02$} \\
\hline & \multicolumn{2}{|c|}{ stellar parameters } \\
\hline & Primary & Secondary \\
\hline & \multicolumn{2}{|c|}{ fractional radii $^{d}$} \\
\hline$\overline{r_{\text {pole }}}$ & $0.07646 \pm 0.00053$ & $0.15703 \pm 0.00055$ \\
\hline$r_{\text {side }}$ & $0.07646 \pm 0.00053$ & $0.15838 \pm 0.00055$ \\
\hline$r_{\text {point }}$ & $0.07646 \pm 0.00053$ & $0.16203 \pm 0.00065$ \\
\hline \multirow[t]{2}{*}{$r_{\text {back }}$} & $0.07646 \pm 0.00053$ & $0.16130 \pm 0.00063$ \\
\hline & \multicolumn{2}{|c|}{ absolute stellar parameters } \\
\hline$\overline{M^{d}\left(\mathrm{M}_{\odot}\right)}$ & $0.567 \pm 0.138$ & $0.177 \pm 0.051$ \\
\hline$R^{d}\left(\mathrm{R}_{\odot}\right)$ & $0.0955 \pm 0.0077$ & $0.1985 \pm 0.0159$ \\
\hline$T_{\text {eff }}($ spec $)(\mathrm{K})$ & $55000 \pm 3000$ & $4000_{-1500}^{+1000}$ \\
\hline$T_{\text {eff }}($ phot $)(\mathrm{K})$ & $55000( \pm 3000)$ & $4648 \pm 55( \pm 259)$ \\
\hline$L_{\mathrm{bol}}^{d}\left(\mathrm{~L}_{\odot}\right)$ & $73.692 \pm 11.819( \pm 19.955)$ & $0.016 \pm 0.004( \pm 0.007)$ \\
\hline $\log g(\operatorname{spec})(\operatorname{cgs})$ & $5.97 \pm 0.30$ & $4.9 \pm 0.5$ \\
\hline \multirow[t]{2}{*}{ 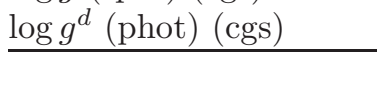 } & $6.23 \pm 0.04$ & $5.11 \pm 0.07$ \\
\hline & \multicolumn{2}{|c|}{ chemical abundances from spectroscopy } \\
\hline$\overline{\log (n \mathrm{He} / n \mathrm{H})}$ & $-2.24 \pm 0.4$ & $-2.4>$ \\
\hline $\log (n \mathrm{C} / n \mathrm{H})$ & -6.0 & $-2.0>$ \\
\hline $\log (n \mathrm{~N} / n \mathrm{H})$ & -6.0 & $-5.0>$ \\
\hline \multirow[t]{2}{*}{$\log (n \mathrm{O} / n \mathrm{H})$} & -6.0 & $-3.5>$ \\
\hline & \multicolumn{2}{|c|}{ flux ratio from spectroscopy } \\
\hline \multirow[t]{2}{*}{$\left(\mathrm{F}_{\mathrm{dM}} / \mathrm{F}_{\mathrm{sd}}\right)_{\max }$ at $4500 \AA$} & \multicolumn{2}{|c|}{$0.43 \pm 0.06$} \\
\hline & \multicolumn{2}{|c|}{ atmospheric model-dependent parameters } \\
\hline$\overline{x_{\text {bol }}}$ & 0.231 & $0.300 \pm 0.01$ \\
\hline$y_{\mathrm{bol}}$ & 0.148 & - \\
\hline$A$ & 1.0 & $1.09 \pm 0.02$ \\
\hline$\beta$ & 1.0 & $2.80 \pm 0.70$ \\
\hline$x_{i}$ & 0.160 & $0.47 \pm 0.02$ \\
\hline$y_{i}$ & 0.108 & - \\
\hline$L_{i} /\left(L_{1}+L_{2}\right)(i)$ & 0.827 & 0.173 \\
\hline$x_{r}$ & 0.184 & 0.50 \\
\hline$y_{r}$ & 0.122 & - \\
\hline$L_{i} /\left(L_{1}+L_{2}\right)(r)$ & 0.986 & 0.014 \\
\hline$x_{R_{C}}$ & 0.179 & $0.50 \pm 0.02$ \\
\hline$y_{R_{C}}$ & 0.119 & - \\
\hline$\underline{L_{i} /\left(L_{1}+L_{2}\right)\left(R_{C}\right)}$ & 0.984 & 0.016 \\
\hline
\end{tabular}

Notes. (1) Parameters without uncertainties were kept fixed, or adopted from precomputed tables. (2) Parameters subscripted with $d$ are derived parameters. (3) Second uncertainties in parentheses were calculated setting the uncertainty of the (fixed) primary effective temperature to be $\delta T_{\text {eff } 1}=3000 \mathrm{~K}$ (i.e. its spectrocopic uncertainty). (4) $x, y, A$ and $\beta$ denote linear and logarithmic limb darkening coefficients, bolometric albedos and gravity brightening exponents, respectively. (5) In the case of the passband-dependent fractional luminosities $\left[L_{i} /\left(L_{1}+L_{2}\right)\right]$, the reflection/irradiation effect was taken into account. 
of a PN around J0640+3856 also supports the evolved hot subdwarf (post-sdB) scenario.

We have constructed a simple model to reproduce the spectral contribution of the irradiated companion. Although this model is optimized for the substellar point, it represents the day side of the companion well, suggesting that the strong irradiation heats up the entire inner hemisphere homogeneously.

Our results suggests that the secondary component may be inflated by only a few percent, like in the cases of similar close binaries (Afsar \& İbanoğlu 2008). We estimate that the substellar point of the red dwarf is heated to about $22500 \mathrm{~K}$. The heat transport of these inflated stars is ineffective, so the large temperature difference between the day and night sides is preserved over long timescales (Ritter, Zhang \& Kolb 2000).

We conclude that the most probable companion spectral type is mid-M. A later-type or more compact companion would be unable to reproduce the eclipses while an earlier type and more massive companion would be inconsistent with the radial velocity curve.

The biggest advantage of J0640+3856 is that it is a double-lined spectroscopic binary. Spectroscopic observations covering the full orbital cycle will yield more precise parameters (especially masses) for the components, as well as an opportunity to monitor and analyse the changing features in the spectra caused by the reflection effect.

The J0640+3856 system is a good analogue to study interactions in planetary systems with hot Jupiters. Both the illumination effect in the primary minimum and the thermal radiation and reflected light disappearance and reappearance in the secondary minimum, is similar as is the luminosity ratio.

The newly discovered J0640+3856 is a unique laboratory in several aspects and opens opportunities to make further steps to understand the evolutionary history of post-common envelope binaries.

This project has been supported by the Hungarian OTKA Grants K83790, K104607, K109276, K113117, ESA PECS Contract No. 4000110889/14/NL/NDe, the Lendület-2009 and the Lendület LP2012-31 Young Researchers Programme of the Hungarian Academy of Sciences and the European Community's Seventh Framework Programme (FP7/2007-2013) under grant agreement no. 269194 (IRSES/ASK) and no. 312844 (SPACEINN). AD has been supported by the Postdoctoral Fellowship Programme of the Hungarian Academy of Sciences and the János Bolyai Research Scholarship of the Hungarian Academy of Sciences. PN was supported by the Deutsche Forschungsgemeinschaft under grant He 1356/49-2. JS acknowledges financial support from STFC in the form of an Advanced Fellowship. TB would like to thank City of Szombathely for support under Agreement No. S-11-1027. Based on observations made with the Gran Telescopio Canarias (GTC), instaled in the Spanish Observatorio del Roque de los Muchachos of the Instituto de Astrofsica de Canarias, in the island of La Palma.

Facilities: WHT, GTC.

\section{REFERENCES}

Afşar, M., \& İbanoğlu, C., 2008, MNRAS, 391, 802

Aller, A., Miranda, L. F., Olguín, L., et al. 2015, MNRAS, 446, 317

Almeida, L. A., Jablonski, F., Tello, J., \& Rodrigues, C. V., 2012, MNRAS, 423, 478

Barman, T. S., Hauschildt, Peter H., \& Allard, F., 2004, ApJ, 614, 338

Benn, C., Dee, K., Agócs, T., 2008, Ground-based and Airborne Instrumentation for Astronomy II. Edited by McLean, Ian S.; Casali, Mark M. Proceedings of the SPIE, 7014, 6

Borkovits, T., Derekas, A., Kiss, L. L., et al., 2013, MNRAS, 428, 1656

Borkovits, T., Derekas, A., Fuller, J., et al., 2014, MNRAS, 443, 3068

Borkovits, T., Rappaport, S., Hajdu, T., Sztakovics, J., 2015, MNRAS, 448, 946

Castelli, F., \& Kurucz, R. L., 2004, arXiv:astro-ph/0405087

Exter, K. M., Pollacco, D. L., Maxted, P. F. L., Napiwotzki, R., \& Bell, S. A. 2005, MNRAS, 359,315 
Ferguson, D. H., Liebert, J., Haas, S., Napiwotzki, R., James, T. A., 1999, ApJ, 518, 866

Günther, H. M., \& Wawrzyn, A. C. 2011, A\&A, 526, AA117

Haefner, R.; Fiedler, A.; Butler, K.; Barwig, H., 2004, A\&A, 428, 181

Han, Z., Podsiadlowski, P., Maxted, P. F. L., Marsh, T. R., \& Ivanova, N., 2002, MNRAS, 336,449

Han, Z., Podsiadlowski, P., Maxted, P. F. L., \& Marsh, T. R., 2003, MNRAS, 341, 669

Heber, U., 2009, ARA\&A, 47, 211

Hilditch, R. W., Kilkenny, D., Lynas-Gray, A. E., \& Hill, G., 2003, MNRAS, 344, 644

Hubeny, I., \& Lanz, T., 1995, ApJ, 439, 875

Kawka A., Vennes S., O’Toole S. et al., 2015, MNRAS, in press

Lanz, T., \& Hubeny, I., 2003, ApJS, 146, 417

Maxted, P. F. L., Heber, U., Marsh, T. R., \& North, R. C., 2001, MNRAS, 326,1391

Maxted, P. F. L., Napiwotzki, R., Dobbie, P. D., \& Burleigh, M. R. 2006, Nature, 442, 543

Mihalas, D. 1978, Stellar Atmospheres, 2nd edn. (San Francisco: Freeman)

Napiwotzki, R., Karl, C. A., Lisker, T., Heber, U., Christlieb, N., Reimers, D., Nelemans, G., \& Homeier, D., 2004, Ap\&SS, 291, 321

Németh, P., Kawka, A., \& Vennes, S. 2012, MNRAS, 427, 2180

Paczynski, B., 1976, Structure and Evolution of Close Binary Systems, 73, 75

Pollacco, D. L., \& Bell, S. A., 1994, MNRAS, 267, 452

Pollacco, D. L., Bell, S. A., \& Hilditch, R. W., 1994, MNRAS, 270, 449

Prša, A., 2011, PHOEBE Scientific Reference, Dept. of Astronomy and Astrophysics, Villanova University
Prša, A., \& Zwitter, T., 2005, ApJ, 628, 426

Ritter, H., Zhang Z.-Y., Kolb U., 2000, A\&A, 360, 969

Savitzky, A., \& Golay, M. J. E. 1964, Analytical Chemistry, 36, 1627

Schlafly, E. F., \& Finkbeiner, D. P. 2011, ApJ, 737,103

Southworth, J., Zima, W., Aerts, C. et al., 2011, MNRAS, 414, 2413

Stroeer, A., Heber, U., Lisker, T., Napiwotzki, R., Dreizler, S., Christlieb, N., \& Reimers, D., 2007, A\&A, 462, 269

Taam, R. E., \& Ricker, P. M. 2006, arXiv:astro-ph/0611043

Vučković, M., Østensen, R., Bloemen, S., Decoster, I., \& Aerts, C., 2008, Hot Subdwarf Stars and Related Objects, 392, 199

Wawrzyn, A. C., Barman, T. S., Günther, H. M., Hauschildt, P. H., \& Exter, K. M. 2009, A\&A, 505,227

Werner, K. 1996, ApJ, 457, L39

This 2-column preprint was prepared with the AAS IATEX macros v5.2. 